\title{
A Combined Approach for Home Automation using Raspberry Pi
}

\author{
R. Josphineleela*, S. S. Sivanya, S. Varshitha and S. Vizhi Arasi \\ Department of Information Technology, Panimalar Institute of Technology, Chennai - 600123, Tamil Nadu, India; \\ pecleela2005@gmail.com,sivanya905@gmail.com,varshitha95@gmail.com, vizhiarasi2@gmail.com
}

\begin{abstract}
In this project, we propose the system for smart home automation using Fuzzy logic with Wi-Fi. It has the capability to perform intelligently adaptive to user preferences, which are focused on improved user comfort, safety and enhanced energy performance. Internet of Things (IoT) is one of the promising technologies which can be used for connecting, controlling and managing intelligent objects which are connected to Internet through an IP address. Devices such as light switches, power plugs, fans, motor, temperature sensors, humidity sensors, and infrared sensors have been integrated in the system to demonstrate the feasibility and effectiveness of the proposed system. This system can control the home appliances automatically based on the threshold values set by the user as well as be remotely controlled by the user himself.
\end{abstract}

Keywords: Home Automation, Internet of Things (IOT), Passive Infra-red Sensors, Raspberry Pi, Remote Access, Whatsapp

\section{Introduction}

In a world which is becoming increasingly automated, Home Automation is acquiring more attention from the households around the world. While many industrial facilities have moved to almost fully automated systems, there are only a few houses that are smartly automated, because of the high cost of such systems. Home Automation should be more accessible since it does not require an incredibly advanced technology and can usually be implemented with off-the-shelf devices. In "smart home" the word "smart" means context aware which can be realized using Information and Communication Technology (ICT) and IoT $\stackrel{1}{2}$. As devices get smarter, a lot of effort is aimed at automating many of our day-to-day activities in a relatively easy way ${ }^{2}$.
Main objective of IoT is to manage and control physical objects around us in a more intelligent and meaningful manner and also improve quality of life by providing cost effective living including safety, security and entertainment.

Home automation or sensible home may be delineated as introduction of technology within the home atmosphere to provide ease and protection to its occupants. By using the technology of the Internet of Things, the examination and execution of home automation have got additional leverage ${ }^{1}$. Various wireless technologies which is able to support some sort of remote knowledge transfer, sensing and management like Bluetooth, Wi-Fi and cellular networks are used to enter abundant levels of acumen within the home. Home automation for the older and disabled will offer raised quality of life for people ${ }^{3}$. It

${ }^{*}$ Author for correspondence 
may provide an interface to home appliances or the automation system itself, via telephone line or the internet, to supply management and observance via a smart phone or personal computer. The Internet can be utilized in home automation that offers several decisions from economical use of energy to additional console, protection and safety. Even over great distances the user can monitor and manage their home gate, various appliances and turn on/off the T.V without any human intervention. Despite these advantages, home automation has however received extensive approval and an attention owing to its high significance and complexness ${ }^{4}$.

\section{Related Works}

Though the concept of smart homes is new in India, considerable amount of work has been carried out in other countries, where smart homes are already in place. Kang ${ }^{\underline{5}}$ discusses about acquisition and analysis of sensor data which are going to be used across smart homes. It proposed an architecture for extracting contextual information by analyzing the data acquired from various sensors and provide context aware services. Andreas Kamilaris ${ }^{\underline{6}}$ discusses the need for common standards and protocols for developing sustainable IoT based applications for smart homes. Pranay P. Gaikwad ${ }^{7}$ discusses about challenges and problems arise in smart home systems using IoT and propose possible solutions. In $\stackrel{8}{ }, \mathrm{Wi}-\mathrm{Fi}$ based home automation system is presented. It uses a PC (with built in Wi-Fi card) based web server that manages the connected home devices. The users can manage and control the system locally (LAN) or remotely (internet). The system supports a wide range of home automation devices like power management components and security components.

Using the Internet as medium is most common because it does not have distance limitations unlike Bluetooth or infrared. Also, the Internet is more reliable than GSM and it has a lower latency too.

\subsection{IoT Architecture}

The IoT-based architecture provides high-level flexibility at the communication and information. It is an approach which is relevant in many different environments such as patient monitoring system, security, traffic signal control or controlling various applications ${ }^{1}$. The IoT project aims to bring out the various opportunities of using IPv6 and other related standards to overcome the disadvantages using of the Internet of Things?. The IoT projects proves a dominant and thorough study of all sensible functionalities, mechanisms and various protocols that can be used for building IoT architectures however interconnections may occur between all totally different IoT applications.

As in the networking field, where several solutions emerged at his infancy to leave place to a common model, the TCP/IP protocol suite, the emergence of a common reference model for the IoT domain and the identification of reference architectures can lead to a faster, more focused development and an exponential increase of IoTrelated solutions ${ }^{\underline{1}}$. These solutions can provide a strategic advantage to mature economies, as new business models can leverage those technological solutions providing room for economic development.

\section{Proposed System}

Every user who is experienced in the existing system may think of a system that may add more flexibility and run with some common applications such as android. This work is designed in such a way to avoid the disadvantages of the existing system. The proposed system supports more elasticity, comfort capacity and safety ${ }^{10}$.

The main objective is to design and to execute a cost effective and open source home automation system that's capable of leading most of the home and sustain the house automation system. The block diagram of the proposed system is shown in Figure 1. The PIR sensor, temperature sensor, humidity sensor, level sensor are used for continuous monitoring of the conditions. Raspberry Pi controller is used as the central component of the system.

In the automated system, when any of the sensor readings exceeds the threshold values, the appliances such as light, fan, and water motor will be automatically switched on/off. This contributes to energy efficiency as it cuts down wastage. 


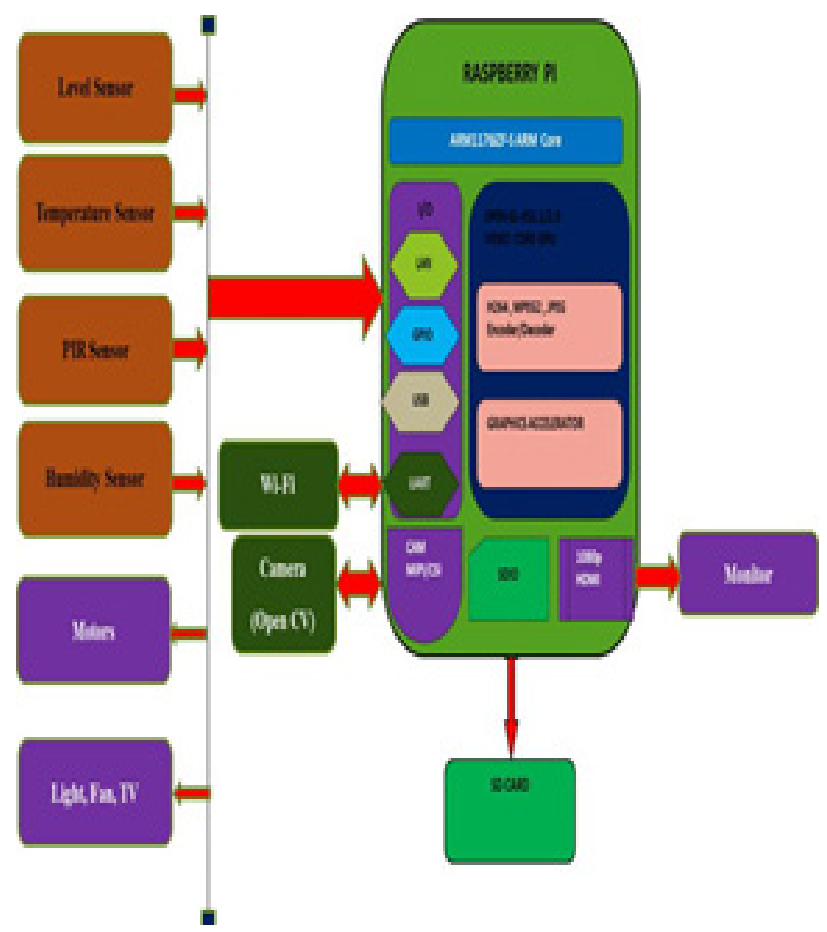

Figure 1. Raspberry Pi controller is used as the central component of the system.
The PIR sensor (Passive Infrared Sensors) detects any movement near the door such as a human entry and triggers the camera interfaced with it. The camera captures the image. The Raspberry Pi controller through its inbuilt Wi-Fi module transmits the image to the owner through Whatsapp.

The owner/user at any remote location can log on to a server through the internet and send control signals to the Raspberry Pi controller, thus controlling the home appliances from a remote location.

This can be used to open the door, switch on/off the lights, fan, TV, etc. The values measured by the sensors can also be monitored from this remote server.

\subsection{Raspberry Pi}

The Raspberry Pi is a low cost credit card sized singleboard computer developed by raspberry pi foundation. Raspberry pi is controlled by a modified version of Debian Linux optimized for the ARM architecture. The core of

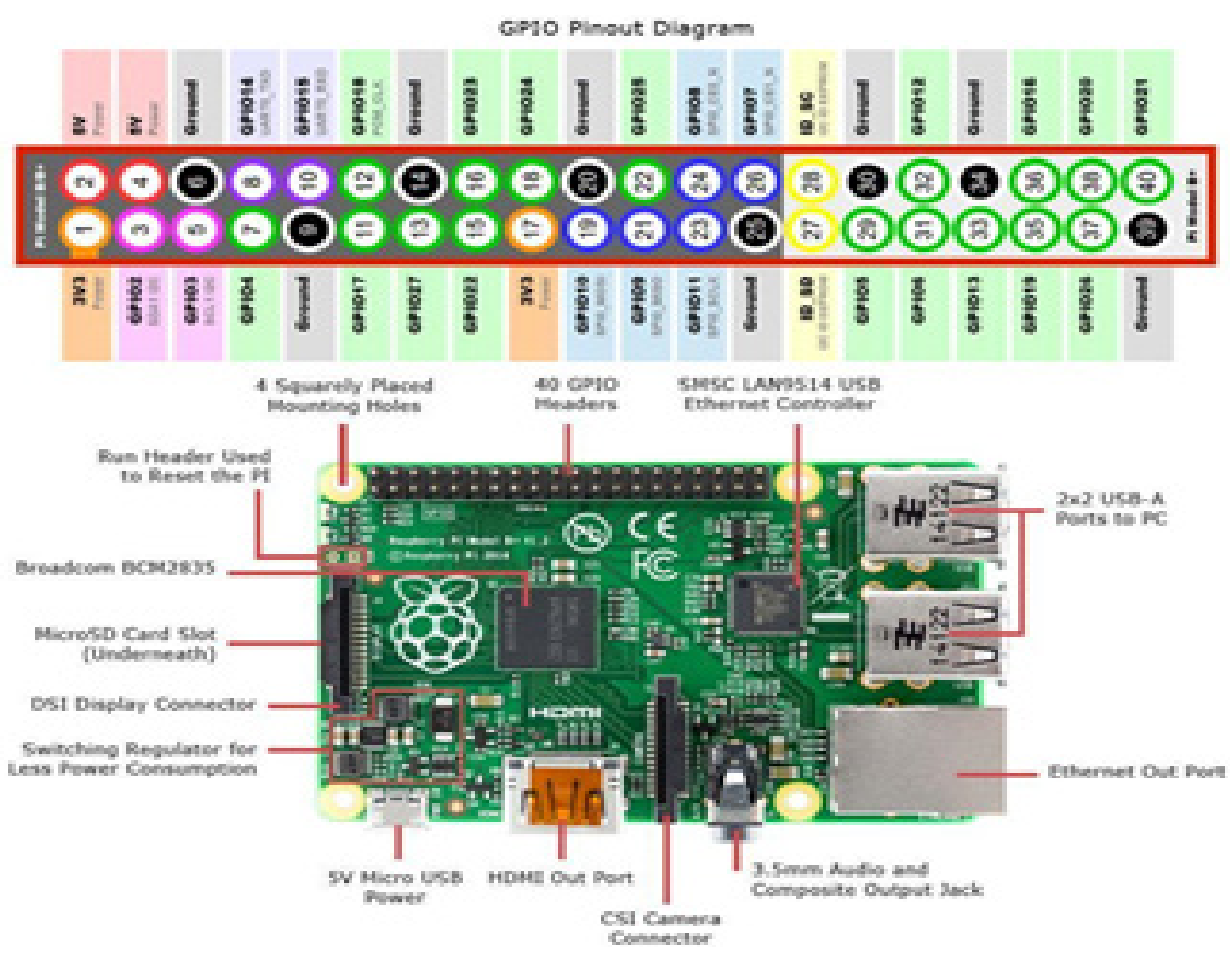

Figure 2. Raspberry Pi controller. 
the home automation system is this minicomputer ${ }^{11}$. Here we are using model B plus. The setting up of raspi consists of selecting raspbian OS from prebuilt SD card. The prebuilt SD card consists of raspbian, arclinux, pidora, open ELEC, risc OS operating system. After the OS selection we need to configure raspberry-pi using Raspi-config command $\frac{10}{}$. We can enter into raspi desktop using startx command.

\subsection{PIR Sensor}

Passive Infrareds sensors (IRs) are electronic devices which are used in some security alarm systems to detect motion of an infrared emitting source, usually a human body.

The pyro electric sensor is made of a crystalline material that generates a surface electric charge when exposed to heat in the form of infrared radiation. When the amount of radiation striking the crystal changes, the amount of charge also changes and can then be measured with a sensitive FET device built into the sensor. This radiation (energy) is invisible to the human eye but can be detected by electronic devices designed for such a purpose sensor values are transmitted by the controller through its Wi-Fi module which can be monitored and controlled from a remote web server.

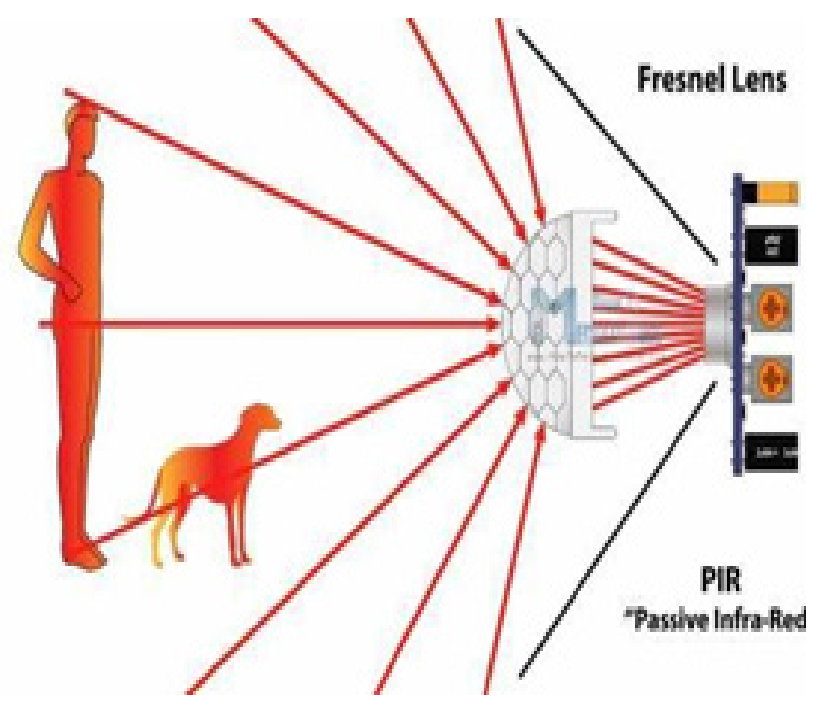

Figure 3. PIR sensor.

\subsection{Wi-Fi Router Configuration}

The wifi unit provides the medium for communication. It can be also configured to make security services. The wifi should be configured with a certain address and user commands will be directing through wifi unit. We may use sudo nano/etc/network/interfaces for configuring wifi with raspberry-pi.

\subsection{Web Server}

Various applications located at home can be remotely controlled or monitored by implanting the devices with the web server. The static and dynamic information are stored in embedded system and it fulfills the demands on web browsers. Such type of web servers are called embedded web server ${ }^{12}$. It's not solely that we will use the Raspberry Pi to induce the information from servers via the web; however it also can act as a server itself. There are many alternative web servers that may be installing on the Raspberry Pi. Ancient web servers, like Apache, serve the files from Raspberry pi board to purchasers. Raspberry pi also can serve sound, video, workable programs, and far a $\operatorname{lot}^{\frac{13}{}}$. However, there's a new breed of tools that reach programming languages like Python, Ruby, and JavaScript to make net servers that dynamically generate the hypertext mark-up language once they receive communications protocol requests from an online browser.

\subsection{Proposed Architecture}

The proposed IoT based architecture has two streamlines of working: (1) Automated control based on threshold values and also monitoring the status and control from a remote location (2) Notification to the user in case of an intruder and prompting them to control the system working.

The first method of working is depicted in Figure 3 which shows how the Raspberry Pi controls the home appliances based on the input from various sensors. The sensor values are transmitted by the controller through its WiFi module which can be monitored and controlled from a remote web server. 


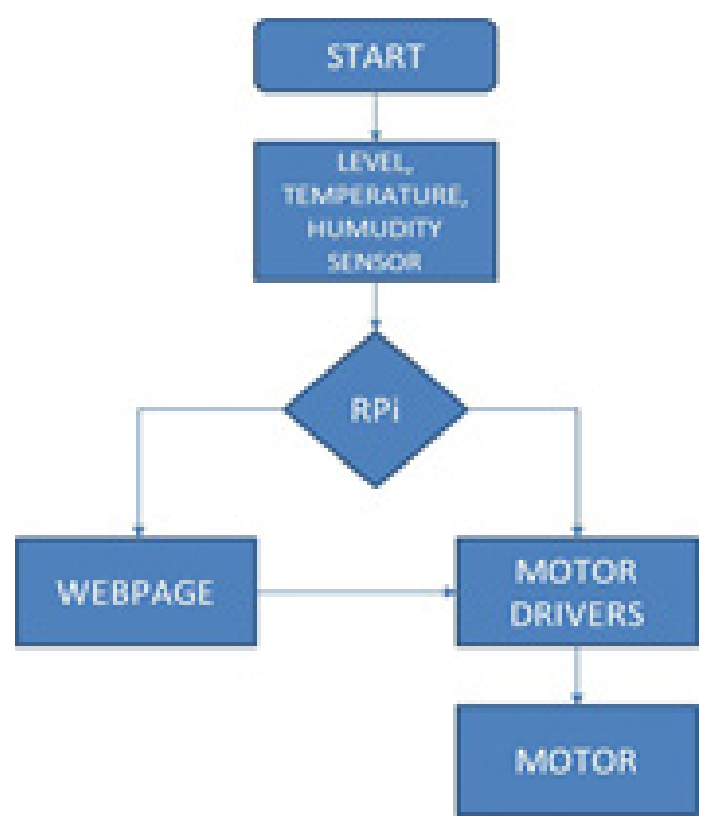

Figure 4. Working of the system.

The second method of working is shown in Figure 4 which depicts how the PIR sensor is triggered upon any

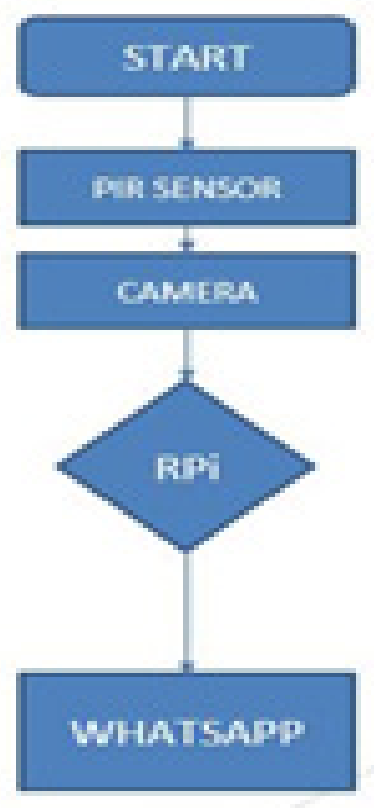

Figure 5. Working of the system when triggered by the PIR sensor. intrusion in the locality of the sensor which in turn signals the camera that has been interfaced with it to capture an image. The Raspberry Pi controller transmits this image with the help of Wi-Fi to the user/owner on his Whatsapp from where the user may decide on how to control the system.

\section{Conclusion and Further Enchancement}

The system proposed in this paper is aimed at automating the use of some of the home appliances such as light, fan, TV and water motor and at the same time controlling from a remote web server with the help of a Raspberry Pi controller. The PIR sensor module can be viewed as a security measure for the home as well as be used by VIPs when they want to grant access to other people in case of necessities.

The proposed system can find applications in everyday home use, industry applications, as an aid to disabled people and bed-ridden patients and also VIPs.

The future enhancements could be in terms of enhancing the security by encrypting the data to provide data security and also from various attacks by implementing in the cloud infrastructure.

\section{References}

1. Kumar Mandula, Ramu Parupalli, Murty CHAS, Magesh E, Rutul Lunagariya. "Mobile based Horne Automation using Internet of Things (IoT)”. 2015 International Conference on Control, lnstrumentation, Communication and Computational Technologies (ICCICCT), 2015 IEEE 9781-4673-9825-1.

2. Kim Baraka, Marc Ghobril, Sami Malek, Rouwaida Kanj, Ayman Kayssi. "Low cost Arduino/Android-based Energy-Efficient Home Automation System with Smart Task Scheduling". Fifth International Conference on Computational Intelligence, Communication Systems and Networks. 2013. Crossref.

3. Atzori Luigi, Antonio Iera and Giacomo Morabito. "The internet of things: A survey”. Computer Networks. 2010; 54(15):2787-2805.I.S.

4. Rionel Belen Caldo, Derrick Castillo, Joseph T Seranilla, Jose Maria T Castillo, Ivan Carlo C Largo, Marie Antonette T 
Latayan, Michael gbb Gabat. "Development of Wi-Fi based switch control system for home appliances using android phone". 8th IEEE International Conference Humanoid, Nanotechnology, Information Technology. 2015. PMCid:PMC4505052.

5. Byeongkwan Kang, Sunghoi Park, Tacklim Lee and Sehyun Park. "IoT based Monitoring System using Tri-level Context Making Model for Smart Home Services”. 2015 IEEE International Conference on Consumer Electronics (ICCE). 2015. Crossref.

6. Andreas Kamilaris, Andreas Pitsillides. "Towards Interoperable and Sustainable Smart Homes". Proceedings, Paul Cunningham and Miriam Cunningham (Eds) IIMC International Information Management Corporation. 2013.

7. Pranay P Gaikwad, Jyotsna P Gabhane, Snehal S Golait. "A Survey based on Smart Homes System Using Internet-ofThings". 2015 International Conference on Computation of Power, Information and Communication. 2015.

8. ElShafee and Hamed KA. "Design and Implementation of a WiFi Based Home Automation System". World Academy of Science, Engineering and Technology. 2012; 68:2177-80.
9. Kelly Sean Dieter Tebje, Nagender Kumar Suryadevara and Subhas Chandra Mukhopadhyay. "Towards the implementation of IoT for environmental condition monitoring in homes". Sensors Journal. IEEE 2013; 13(10):3846-53. Crossref.

10. Pavithra D, Ranjith Balakrishnan. "IoT based Monitoring and Control System for Home Automation". Proceedings of 2015 Global Conference on Communication Technologies (GCCT 2015), IEEE 2015: 978-1-4799-8553-1.

11. Chowdhury Md Nasimuzzaman, Md Shiblee Nooman and Srijon Sarker. "Access Control of Door and Home Security by Raspberry Pi Through Internet”. International Journal of Scientific and Engineering Research. 2013; 4.

12. Gubbi Jayavardhana et al. "Internet of Things (IoT): A vision, architectural elements, and future directions". Future Generation Computer Systems. 2013; 29(7):164560. Crossref.

13. Fang Shifeng et al. "An Integrated System for Regional Environmental Monitoring and Management Based on Internet of Things". IEEE Trans. Industrial Informatics 2014; 10(2):1596-1605. Crossref. 